\title{
Pleural aspergillosis in a 14 year old boy
}

\author{
M CLIVE KEARON, JOAN T POWER, A E WOOD, LUKE J CLANCY \\ From the Department of Respiratory Medicine and Thoracic Surgery, St James's Hospital and the Royal City \\ of Dublin Hospital, Dublin
}

Pleural aspergillosis is rare, usually occurring in seriously ill adults against a background of tuberculosis and chronic bronchopleural fistulas. ${ }^{1}$ We present a case occurring in a child that differs in many respects from previously reported cases.

\section{Case report}

A 14 year old boy complained of malaise, anorexia, a dry cough, and right sided chest discomfort of three weeks' duration. He was referred for a chest radiograph, which showed a large right sided hydropneumothorax with cavitation at the apex of the right lung. The chest radiograph taken after insertion of a chest drain is shown in figure 1 . He had been in good health until aged 11 years, when two weeks after BCG vaccination he was admitted to hospital with a severe right sided pneumonia with associated cavitation, abscess formation, and a small pleural effusion. At that time routine cultures, Ziehl-Neelsen stains, and culture for Mycobacterium tuberculosis were negative. A presumptive diagnosis of staphylococcal pneumonia was made and he responded to ampicillin and cloxacillin; pleural intubation was not performed. His sweat sodium concentration was normal. He was well during the next 18 months, serial radiographs showing residual pneumatocoeles in the right upper lobe and apical segment of the right lower lobe. During the next two years, after discharge from regular attendance at the paediatric clinic, he had remained reasonably well.

On examination he was thin and pale, but afebrile and in no acute distress. His trachea was deviated to the left and there was stony dullness on percussion over the right mid and lower chest zones. General examination revealed no other abnormality. The haemoglobin concentration was $13 \mathrm{~g} / \mathrm{dl}$ and the white cell count $7.4 \times 10^{9} / 1$ with a normal differential count. A Mantoux test gave a strongly positive reaction at 1 TU strength. Fluid removed by pleural aspiration was cloudy, cytological examination showing $85 \%$ polymorphonuclear leucocytes and no malignant cells. The protein concentration was $36 \mathrm{~g} / \mathrm{l}$. Gram stains, Ziehl-Neelsen stains, and cultures for pyogenic organisms and for $M$ tuberculosis from various sites were repeatedly negative. Closed pleural biopsy showed histiocytes but no granulomas, fungi, or acid fast bacilli. No fungal elements were

Address for reprint requests: Dr Luke Clancy, TCD Medical School, St James's Hospital, Dublin 8. seen in the empyema pus and there was no fungal growth on culture.

Despite wide bore intercostal drainage, suction, irrigation, and conventional antibiotic and antituberculous chemotherapy, the right hydropneumothorax failed to resolve satisfactorily. Bronchography showed filling of some of the cavities with contrast medium; no bronchiectasis or bronchopleural fistula was demonstrated, although a slow air leak continued. On the 35 th hospital day a right thoracotomy was performed with evacuation of $450 \mathrm{ml}$ of pus from a large empyema cavity. Pleural decortication was also undertaken; the resected pleura was grossly thickened to a width of $2 \mathrm{~cm}$. Histological examination of the pleura showed necrotising granulomas with multinucleate giant cells (fig 2). The central necrotic material contained fungal hyphae with appearances consistent with aspergillus. Stains for mycobacteria were negative. The appearances were consistent with a healing empyema and were uniform throughout the numerous sections that were made. Postoperatively the patient was treated with intravenous amphotericin $(250 \mu \mathrm{g} / \mathrm{kg}$ a day for 21 days, total dose $236 \mathrm{mg})$ and made an

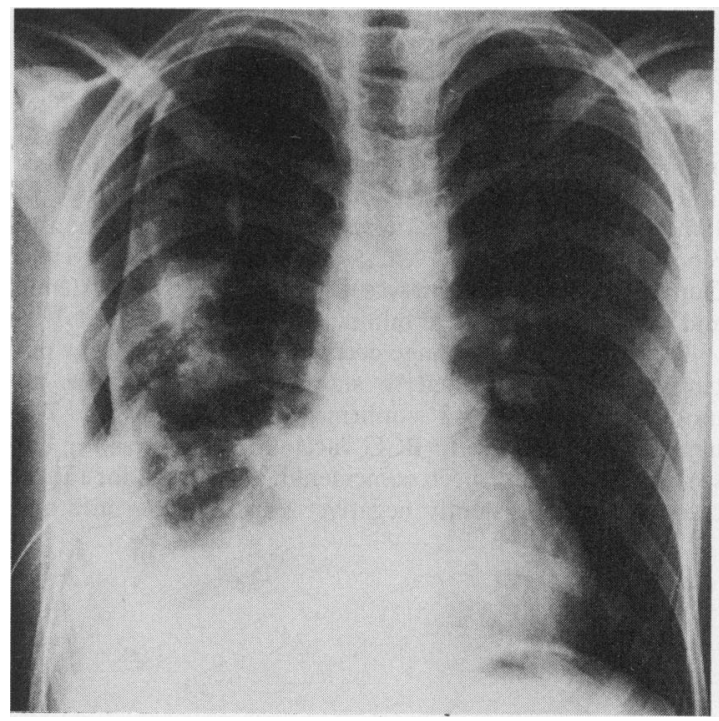

Fig 1 Chest radiograph showing a right pyopneumothorax with cavitation in the apex of the right lung; a right intercostal drain is in situ. 


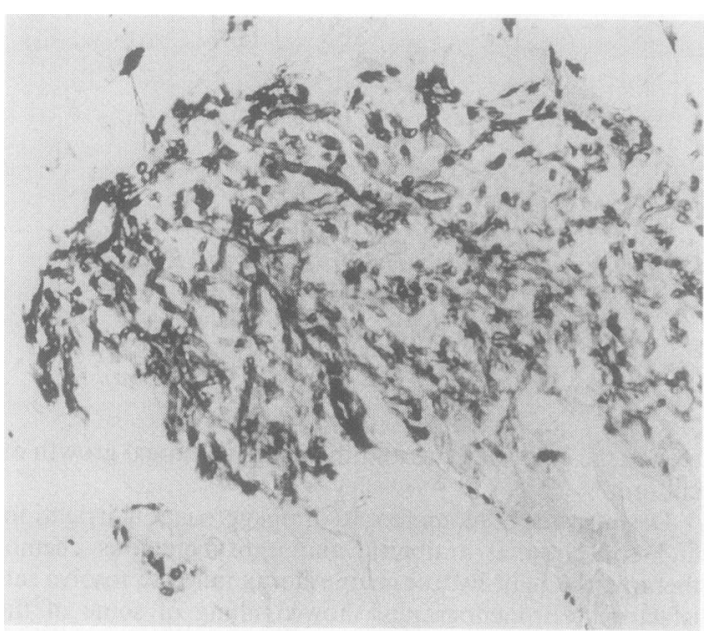

Fig 2 Histological appearance of the resected pleura showing the presence of invasive fungi consistent with aspergillus (Grocott stain).

uneventful recovery. He was discharged from hospital on his 67th hospital day and continues to be well without relapse six months later.

Investigations of the boy's immune state, including white cell count and differential, serum immunoglobulin and complement concentrations, neutrophil chemiluminescence, and lymphocytes, gave normal results. Testing for precipitins to Aspergillus fumigatus gave a strongly positive result.

\section{Discussion}

Pleural aspergillosis is rare, with less than 30 reported cases. ${ }^{1-3}$ Unlike invasive pulmonary aspergillosis, it has not been associated with systemic immunosuppression, but local pulmonary defence mechanisms are frequently disrupted. Of the 26 cases reported since $1957,77 \%$ had active or treated tuberculosis and $65 \%$ had bronchopleural fistulas. Pleural intubation had been performed in half the cases, and infection of the pleura secondary to lung resection occurred in $19 \%$. In contrast to our patient, the mean age of previously reported patients was 53 years, the youngest being 27 years. Our patient's presentation was also atypical in that systemic and local symptoms were minimal.

The original lung damage occurred at 11 years in our patient and was attributed to staphylococcal infection, although this was never confirmed bacteriologically. The temporal relationship to BCG vaccination was striking but seems likely to have been coincidental as cultures for tuberculosis were consistently negative. Furthermore, only one case of metastatic pulmonary BCG infection has been re-: ported worldwide and this patient, unlike our own, showed evidence of impaired cellular immunity. ${ }^{4}$ We believe thato there was direct invasion of the pleura from the lung, which $\frac{\bar{\sigma}}{5}$ had been previously damaged by infection and later pre- $\overline{\frac{5}{5}}$ sumably superinfected with aspergillus fungus. Possibly this $\vec{\nabla}$ spread to the pleura occurred by means of the pneu- $\varrho$ mothorax, but in view of the thickening of the pleura and the absence of fungi in the pleural aspirates direct invasion from lung parenchyma seems more likely.

Whereas the more common invasive aspergillosis found in $\overrightarrow{\mid \vec{\omega}}$ association with immunosuppression is associated with negative results in aspergillus precipitin tests, ${ }^{56}$ in all eight cases of pleural aspergillosis, including our own, in which $\stackrel{\times}{x}$ precipitins have been estimated results have all been positive. $\hat{N}$ This reflects the immune competence shared by this group of patients.

The best treatment for pleural aspergillosis remains un- $\checkmark$ clear because of the small number, widely differing treat-음 ments, and differing conditions of previously reportedpatients. If an early diagnosis is made, a trial of pleural $c$ drainage and intravenous amphotericin can be undertaken. Our patient had a complicated empyema with failure of the right lung to re-expand. A surgical approach with $\vec{\odot}$ decortication and full reinflation of the affected lung was the $\stackrel{\infty}{-}$ only approach that would have been effective. Because of the presence of invasive aspergillus in all sections of the resected pleura we believed that a course of parenteral antifungal chemotherapy was mandatory.

The occurrence of pleural aspergillosis is not confined to the elderly, debilitated, and critically ill. It should be consid- $\stackrel{\mathbb{Q}}{\Omega}$ ered in patients with pleural disease of unknown aetiology, $\overrightarrow{\vec{O}}$ when the presence of serum precipitins against aspergillus 3 can be a helpful diagnostic pointer.

We thank Dr Sally Bonar, Department of Pathology, and Ms Linda Dromgoole for their help and cooperation.

\section{References}

1 Krakowka P, Rowinska E, Halweg $H$. Infection of the Pleura by Aspergillus fumigatus. Thorax 1970;25:245-53.

2 Meredith HC, Cogan BM, McLaulin B. Pleural aspergillosis. Am J Roentgenol 1978;130:164-6.

3 Herring M, Percora D. Pleural aspergillosis: a case report. $\mathrm{Am}$ Surg 1976;42:300-2.

4 Marks J, Jenkins PS, Kilpatrick GS, Engbaek HC, Vergmann B. Pulmonary tuberculosis due to BCG. Br Med J 1971;3:229-30. N

5 Young RC, Bennett JE. Invasive aspergillosis: absence of detectable antibody response. Am Rev Respir Dis 1971; $N$ 104:710-6.

6 Bardana EJ, Gerber JD, Craig S, Cianciulli FD. The general and specific humoral immune response to pulmonary aspergillosis. Am Rev Respir Dis 1975;112:799-805. 